\title{
Lower limit for differential rotation in members of young loose stellar associations ${ }^{\star}$
}

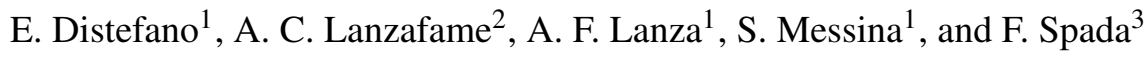 \\ 1 INAF-Osservatorio Astrofisico di Catania, via S. Sofia, 78, 95123 Catania, Italy \\ e-mail: elisa.distefano@oact.inaf.it \\ 2 University of Catania, Astrophysics Section, Dept. of Physics and Astronomy, via S. Sofia, 78, 95123 Catania, Italy \\ 3 Leibniz-Institut für Astrophysik Potsdam (AIP), An der Sternwarte 16, 14482 Potsdam, Germany
}

Received 4 November 2015 / Accepted 5 April 2016

\begin{abstract}
Context. Surface differential rotation (SDR) plays a key role in dynamo models and determines a lower limit on the accuracy of stellar rotation period measurements. SDR estimates are therefore essential to constrain theoretical models and infer realistic rotation period uncertainties.

Aims. We measure a lower limit to SDR in a sample of solar-like stars belonging to young loose stellar associations with the aim of investigating how SDR depends on global stellar parameters in the age range (4-95 Myr).

Methods. The rotation period of a solar-like star can be recovered by analyzing the flux modulation caused by dark spots and stellar rotation. The SDR and the latitude migration of dark-spots induce a modulation of the detected rotation period. We employed longterm photometry to measure the amplitude of such a modulation and to compute the quantity $\Delta \Omega_{\text {phot }}=\frac{2 \pi}{P_{\min }}-\frac{2 \pi}{P_{\max }}$ that is a lower limit to SDR.

Results. We find that $\Delta \Omega_{\text {phot }}$ increases with the stellar effective temperature and with the global convective turn-over timescale $\tau_{\mathrm{c}}$, which is the characteristic time for the rise of a convective element through the stellar convection zone. We find that $\Delta \Omega_{\text {phot }}$ is proportional to $T_{\text {eff }}^{2.18 \pm 0.65}$ in stars recently settled on the ZAMS. This power law is less steep than those found by previous authors, but closest to recent theoretical models. We investigate how $\Delta \Omega_{\text {phot }}$ changes in time in a $\sim 1 M_{\odot}$ star. We find that $\Delta \Omega_{\text {phot }}$ steeply increases between 4 and $30 \mathrm{Myr}$ and that it is almost constant between 30 and $95 \mathrm{Myr}$. We find also that the relative shear increases with the Rossby number Ro. Although our results are qualitatively in agreement with hydrodynamical mean-field models, our measurements are systematically higher than the values predicted by these models. The discrepancy between $\Delta \Omega_{\text {phot }}$ measurements and theoretical models is particularly large in stars with periods between 0.7 and $2 \mathrm{~d}$. Such a discrepancy, together with the anomalous SDR measured by other authors for HD 171488 (rotating in $1.31 \mathrm{~d}$ ), suggests that the rotation period could influence SDR more than predicted by the models.
\end{abstract}

Key words. stars: solar-type - starspots - stars: rotation - open clusters and associations: general - techniques: photometric

\section{Introduction}

Surface differential rotation (SDR) has been detected and measured in a wide sample of solar-like stars (e.g., Barnes et al. 2005; Collier Cameron 2007; Reinhold et al. 2013). These measurements are essential to constrain theoretical models that try to investigate the connections between rotation, convection, and the topology of stellar magnetic fields. The amplitude of SDR influences the type of dynamo operating inside the star. Differential rotation is able to generate a strong toroidal magnetic field out of a weak poloidal field, and is therefore a key ingredient of $\alpha \Omega$ dynamos (e.g., Parker 1955; Brandenburg et al. 1991). On the other hand, stars characterized by a low SDR degree that rotate as solid bodies can instead sustain only an $\alpha^{2}$-type dynamo (e.g., Moss \& Brandenburg 1995; Küker \& Rüdiger 2011).

The amplitude of the SDR is usually measured through the surface rotational shear:

$\Delta \Omega=\Omega_{0}-\Omega_{\text {pole }}$,

* Tables 2-5 are only available at the CDS via anonymous ftp to cdsarc.u-strasbg.fr (130.79.128.5) or via

http://cdsarc.u-strasbg.fr/viz-bin/qcat?J/A+A/591/A43 where $\Omega_{0}$ and $\Omega_{\text {pole }}$ are the surface angular velocities at the equator and the poles, respectively. If $\Delta \Omega>0$, then the star has a solar-like SDR, meaning that the equator rotates faster than the poles. If $\Delta \Omega<0$, the star exhibits an antisolar SDR.

In recent years, several authors investigated how the SDR depends on stellar effective temperature and rotation period. Barnes et al. (2005) reported $\Delta \Omega$ measurements obtained by means of the Doppler imaging technique for a sample of ten young latetype stars. They found that $\Delta \Omega$ is strongly dependent on the stellar temperature $\left(\Delta \Omega \propto T_{\mathrm{eff}}^{8.93 \pm 0.31}\right)$ but poorly correlated to $\Omega_{0}$ $\left(\Delta \Omega \propto \Omega_{0}^{0.15 \pm 0.1}\right)$. A strong correlation between $\Delta \Omega$ and $T_{\text {eff }}$ was also found by Collier Cameron (2007), who enlarged the sample of stars studied by Barnes et al. (2005) and found the relation $\Delta \Omega \propto T_{\text {eff }}^{8.6}$.

The results found by these works are in quantitative disagreement with theoretical models developed by Kitchatinov \& Rüdiger (1999), Küker \& Rüdiger (2011), and by Küker et al. (2011). These theoretical works predict that the SDR increases with the stellar temperature and that it is almost independent of the stellar rotation period. However, the power law expected from these models is $\Delta \Omega \propto\left(\frac{T_{\text {eff }}}{5500 \mathrm{~K}}\right)^{2}$ in the range $3800-6000 \mathrm{~K}$ and $\Delta \Omega \propto\left(\frac{T_{\text {eff }}}{5500 \mathrm{~K}}\right)^{20}$ above $6000 \mathrm{~K}$ (Küker \& Rüdiger 2011). 
Table 1. Stellar associations

\begin{tabular}{llll}
\hline \hline Association & N. & $\begin{array}{l}\text { age } \\
(\mathrm{Myr})\end{array}$ & Ref. \\
\hline$\epsilon$ Chamaleontis ( $\epsilon$ Cha) & 9 & $3-5$ & 1,2 \\
$\eta$ Chamaleontis $(\eta$ Cha) & 4 & $6-10$ & $1,3,4$ \\
TW Hydrae (TWA) & 9 & $8-12$ & 5,6 \\
$\beta$ Pictoris $(\beta$ Pic) & 18 & $12-22$ & 7,8 \\
Octans (Oct) & 1 & $20-40$ & 9,10 \\
Columba (Col) & 14 & $20-40$ & 11,12 \\
$\eta$ Carinae $(\eta$ Car) & 12 & $20-40$ & 11,12 \\
Tucana-Horologium (Tuc/Hor) & 17 & $20-40$ & 11,12 \\
Argus (Arg) & 10 & $30-50$ & $9,13,14$ \\
IC 2391 & 3 & $30-50$ & 13,14 \\
AB Doradus (AB Dor) & 14 & $70-120$ & $15,16,17$ \\
\hline
\end{tabular}

Notes. For each association we report the number of members for which we estimated $\Delta \Omega_{\text {phot }}$, the age estimate, and the age reference.

References. (1) Murphy et al. (2013); (2) Feigelson et al. (2003); (3) Mamajek et al. (1999); (4) Lawson \& Feigelson (2001); (5) Webb et al. (1999); (6) Barrado Y Navascués (2006); (7) Song et al. (2003); (8) Makarov (2007); (9) Torres et al. (2008); (10) Murphy \& Lawson (2015); (11) Torres et al. (2001); (12) Zuckerman \& Webb (2000); (13) De Silva et al. (2013); (14) Barrado y Navascués et al. (2004); (15) Mentuch et al. (2008); (16) López-Santiago et al. (2006); (17) Luhman et al. (2005).

Reinhold et al. (2013) analyzed the Quarter 3 (Q3) longcadence photometric data collected by the Kepler mission (Borucki et al. 2010) and searched for the SDR in a sample of about 20000 main-sequence late-type stars. In contrast with Barnes et al. (2005) and Collier Cameron (2007), they found that the SDR exhibits a weak dependence on temperature in the range $3800-6000 \mathrm{~K}$, and that above $6000 \mathrm{~K}$, it steeply increases toward higher temperatures. Reinhold et al. (2013) claimed that their results, although quite scattered, agree better with the model of Küker \& Rüdiger (2011). We note, however, that these authors have no information on the ages of their targets. This lack of information makes it difficult to compare their results with those of Barnes et al. (2005) and Collier Cameron (2007). The targets investigated by Barnes et al. (2005) and Collier Cameron (2007) comprise pre-main-sequence stars and stars recently settled on the zero-age main sequence (ZAMS), while the sample of Reinhold et al. (2013) included field stars of different ages. Moreover, their data do not permit studying the SDR as a function of the stellar age.

The present work is focused on a sample of 111 late-type stars belonging to 11 nearby young loose associations with known ages (Table 1). The ages of these associations span the interval (4-95 Myr), and therefore our study concerns the SDR evolution during the transition between the pre-main-sequence and the main-sequence phase. We searched for the SDR by analyzing long-term photometric time-series collected by the All Sky Automatic Survey (ASAS, Pojmanski 1997) and the Wide Angle Search for Planets (SuperWASP, Pollacco et al. 2006).

The paper is organized in the following way: in Sect. 2 we describe the photometric data. In Sect. 3 we explain the method we employed to measure the quantity $\Delta \Omega_{\text {phot }}{ }^{1}$, that is, a lower limit for the SDR. In Sect. 4 we investigate how $\Delta \Omega_{\text {phot }}$ depends 1 The subscript stands for photometric and indicates the type of data
used to measure the SDR. on global stellar parameters. In Sect. 5 we discuss the main results of our study, and in Sect. 6 the conclusions are drawn.

\section{Data}

Messina et al. (2010, 2011) analyzed ASAS and SuperWASP time series for about 300 stars belonging to nearby young associations and measured the rotation period for most of these stars. In this work we reprocessed 99 ASAS time series and 31 SuperWASP time series with a new technique (see Sect. 3) for estimating a lower limit $\Delta \Omega_{\text {phot }}$ for the rotational shear. In Table 2 we list the 111 stars studied here together with their color indexes and spectral types. These targets were selected from those studied in Messina et al. $(2010,2011)$ by picking up those for which the largest number of photometric measurements is available. For nine of these targets both ASAS and SuperWASP time series are available. We note that the ASAS time series processed here span an interval time longer than those processed in Messina et al. (2010, 2011).

\subsection{ASAS photometry}

The ASAS time series analyzed in the present work are contained in the ASAS-3 photometric $V$-band catalog ${ }^{2}$. These time series cover a time span of about $13 \mathrm{yr}$ (from 1997 to 2010) and have a typical photometric error of $0.02 \mathrm{mag}$. This error allows the detection of rotational modulation in young solar-like stars where the amplitude modulation ranges between 0.05 and $0.3 \mathrm{mag}$ (Messina et al. 2011). In our analysis we used filtered time series obtained by selecting only the best photometric data. The time-series sampling depends on the star coordinates. We divided the ASAS time series into three groups for which the average interval $t_{\text {mean }}$ between two consecutive observations was $\simeq 1,2$, and $3 \mathrm{~d}$, respectively.

\subsection{SuperWASP photometry}

The SuperWASP time series were downloaded from the first public data archive (Butters et al. 2010). These time series cover a time span of about four years (from 2004 to 2008). Their time coverage is quite irregular and depends on the sky coordinates. The time series processed in the present work are typically made of two or three distinct observational seasons covering 60-300 d intervals. During each season, a target was observed every day for a few hours with a sampling rate of about ten minutes in the most favorable sky positions. The SuperWASP data therefore have a better sensitivity to periods $\leq 1 \mathrm{~d}$ than the ASAS data. Our analysis is based on the processed flux measurements obtained through applying the SYSREM algorithm (Tamuz et al. 2005). SuperWASP observations were collected in 2004 without any light filter. Starting from 2006, they were collected through a wide-band filter in the range 400-700 nm. Owing to differences in spectral bands with respect to the standard Johnson $V$ ASAS data, we analyzed the SuperWASP data independently without merging them with ASAS data.

\section{Method}

The surface of a solar-like star is covered by magnetically active regions (ARs) associated with dark spots and bright faculae. If the star is a differential rotator, the ARs will rotate with different

\footnotetext{
2 http://www . astrouw. edu.pl/asas/
} 
frequencies, depending on their latitudes, and will modulate the optical flux from the star. ARs migration in latitude induces a modulation of the measured photometric period that can be used to estimate the amplitude of SDR. Based on this, the method we employed to measure the SDR on a given star consists of the followings steps:

- we segmented the photometric time-series with a slidingwindow algorithm;

- we searched for the stellar rotation period in each segment;

- we selected the longest and shortest detected periods and computed the quantity $\Delta \Omega_{\text {phot }}=\Omega_{\max }-\Omega_{\min }$, where $\Omega_{\max }=\frac{2 \pi}{P_{\min }}$ and $\Omega_{\min }=\frac{2 \pi}{P_{\max }}$.

We assumed that $P_{\max }$ and $P_{\min }$ are related to time-series segments where the stellar surface is dominated by high-latitude and low-latitude ARs, respectively (or by the opposite configuration for an antisolar SDR). Since the $P_{\max }$ and $P_{\min }$ thus found do not necessarily sample the whole latitude range, $\Delta \Omega_{\text {phot }}$ is obviously a lower limit for the rotational shear and therefore $\Delta \Omega_{\text {phot }} \leq \Delta \Omega$.

\subsection{Time-series segmentation}

Time-series segmentation is very useful in dealing with magnetically active stars. In fact, the typical light curves of these variables are not very regular because the flux variation that is due to rotational modulation can be masked by the intrinsic evolution of ARs.

In the Sun, ARs evolve on a typical timescale $\tau_{\mathrm{AR}} \simeq 60 \mathrm{~d}$ (Lanza et al. 2003). This timescale, which is about twice the solar rotation period, and the simultaneous occurrence of different ARs over the solar disk might in principle destroy the coherence of the rotational signal and prevent the detection of the rotation period. However, ARs in the Sun tend to form at active longitudes, that is, at heliographic longitudes characterized by the frequent localized emergence of new magnetic flux. These AR complexes (ARCs), forming at active longitudes, evolve on a typical timescale $\tau_{\mathrm{ARC}} \simeq 200-250 \mathrm{~d}$ (Lanza et al. 2003). Hence, the rotational signal is approximately coherent on time intervals of the same order as $\tau_{\text {ARC }}$. Lanza et al. (2004) analyzed the total solar irradiance (TSI) time series collected by the SoHo satellite and showed that the use of period-search algorithms to detect the rotation period of the Sun is only possible if the long-term time series is divided into sub-series with a time extension no longer than $150 \mathrm{~d}$ in order to limit the effect of the evolution of ARCs.

The timescales of ARs and ARCs evolution in late-type stars other than the Sun have been studied to a limited extent because of the lack of multi-year continuous photometric time-series. Donahue et al. (1997a,b) estimated the timescales of AR evolution in some tens of late-type stars by analyzing long-term time series of photometric fluxes in two $0.1 \mathrm{~nm}$ passbands centered on the $\mathrm{Ca}$ II $\mathrm{H}$ and $\mathrm{K}$ emission lines. They showed that the older and less magnetically active stars tend to be AR evolutiondominated. This means that the evolution of ARs and ARCs take place on timescales comparable with the stellar rotation period. The light curves of younger and more active stars, conversely, exhibit a pattern that remains stable for several consecutive rotations. The typical timescales measured for these stars are $\tau_{\mathrm{AR}}=50 \mathrm{~d}$ and $\tau_{\mathrm{ARC}}=1 \mathrm{yr}$. Similar values are reported by Hussain (2002). Messina \& Guinan (2003) analyzed long-term photometric time-series collected in the Johnson $V$-band for six young solar analogs. They found that two of these stars are evolution-dominated, while the others have $\tau_{\text {ARC }}$ values ranging between $160 \mathrm{~d}$ and $500 \mathrm{~d}$, remarkably longer than their rotation periods.
The advantage of using time-series segments is that the data exhibit a more stable pattern of flux variations than the whole time-series, but they also contain fewer points. Therefore, the rotation period retrieved by analyzing a time-series segment could have a low statistical significance. A compromise has been found between the length of the segments and the number of points included in the segments by adopting a length $T=50 \mathrm{~d}$. For ASAS data, the average number of points per segment ranges between 16 and 50, depending on the source coordinate. For Super-WASP data, the average number of points per segment ranges between 1000 and 3000 . We note that $T=50 \mathrm{~d}$ is also the typical $\tau_{\mathrm{AR}}$ timescale found by Donahue et al. (1997a) for young active stars. In Sect. 3.5 we discuss the effect of the choosing different segment durations on $\Delta \Omega_{\text {phot }}$ measurements.

We segmented the time series by using a sliding-window algorithm as sketched in Fig. 1. This algorithm performs a cycle on the whole time-series. For each observation time $t_{i}$, a segment is generated by selecting all points in the time interval $\left[t_{i}, t_{i}+T\right]$, where $T$ is the length of the window. A segment is rejected if it has fewer then ten points or if it is a subset of another segment (this last case occurs when the time series exhibits gaps larger than the sliding window; see Fig. 1).

In Fig. 1 we show the $V$-band time series of the star ASAS J070030-7941.8. The observations span about nine years. The red and green bullets mark the data processed by Messina et al. (2010). The different colors are used to display the segmentation performed in that work. The black crosses mark data collected after February 2008 that were not processed by Messina et al. (2010). Messina et al. (2010) extracted ten sub-series. Our sliding window algorithm allows the extraction of more segments.

\subsection{Rotation period and SDR estimate}

We performed the period search by means of the Lomb-Scargle periodogram, which has been found to be a highly efficient method for solar-like variables (Distefano et al. 2012). For each time-series segment we computed the periodogram and selected the period $P$ with the highest power $Z$. We built the distribution of the detected periods and estimated the average stellar rotation period by taking the mode of the distribution.

In the left panel of Fig. 2 we show the frequency histogram obtained for the source ASAS J070030-7941.8. The distribution exhibits a well-defined peak at $P_{\text {rot }}=5.12 \mathrm{~d}$ that is taken as the average stellar rotation period. The other peaks occur at the beat periods $B$ because of the interference between the rotational modulation and the typical ASAS one-day sampling and are given by the relationship $B=P_{\text {rot }} /\left|1 \pm n P_{\text {rot }}\right|$ with $n=1,2,3, \ldots$

After estimating the average rotation period, we again performed the period search by rejecting peaks close to the beat frequencies and by selecting for each segment the highest significant peak closest to the average rotation period. We built the true period distribution and picked up $P_{\min }$ and $P_{\max }$ to compute $\Delta \Omega_{\text {phot }}$. In some cases the period distributions show outliers. These outliers might be related to real rotation frequencies or to false positives and might lead to an overestimate of $\Delta \Omega_{\text {phot }}$. To avoid this, we estimated $P_{\min }$ and $P_{\max }$ by taking the 5 th and 95th percentile of the distribution. We note that this rejection criterion is very cautious. Fewer than $1-2 \%$ of the measurements are distant more than $3 \sigma$ from the average period for most of our targets. In the right panel of Fig. 2 we show the true period distribution obtained for ASAS J070030-7941.8 after beat rejection. We also report the error bars associated with $P_{\min }$ and $P_{\max }$. In Figs. 3-5 we also plot the distributions 


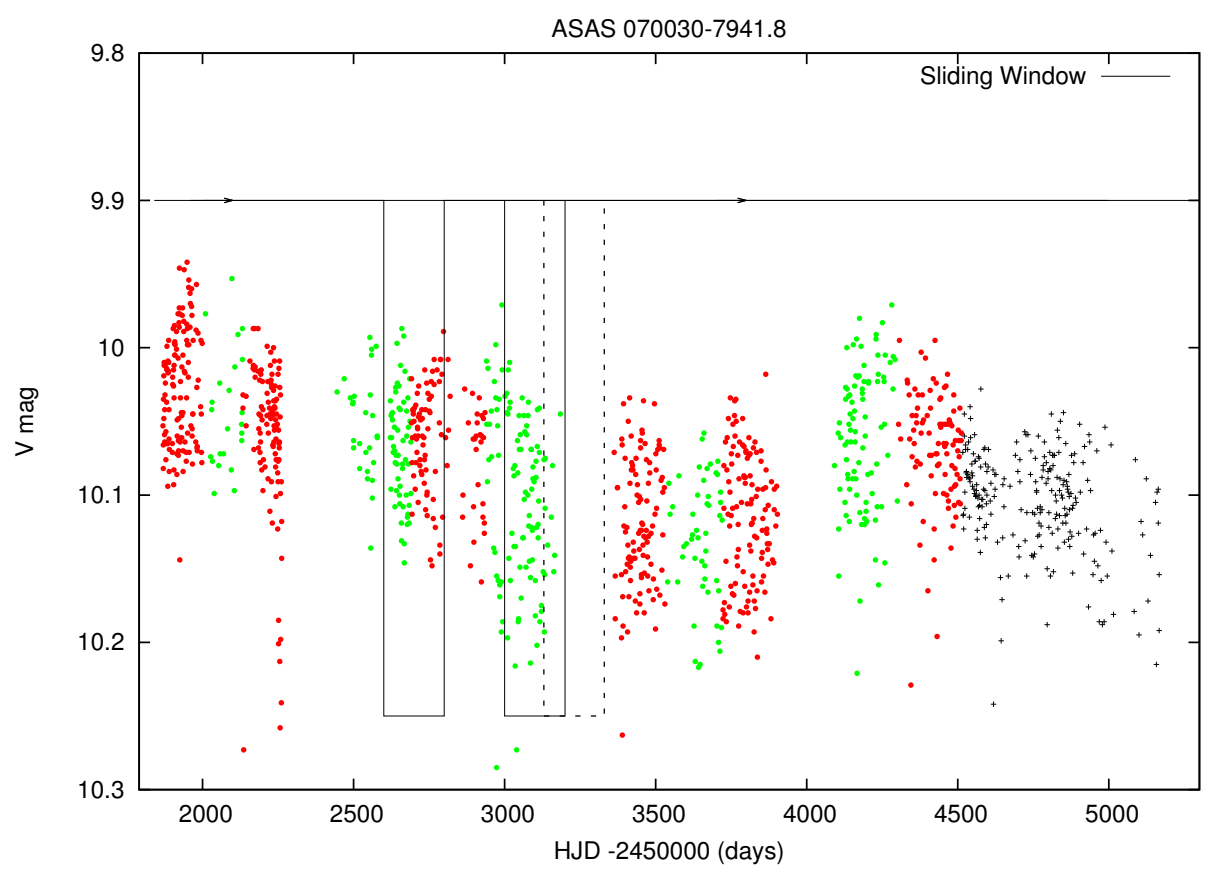

Fig. 1. Typical $V$-band time series collected by the ASAS survey. The red and green bullets mark the segmentation performed by Messina et al. (2010). The black continuous boxes depict two of the extracted segments, while the dotted box indicates one of the sub-series rejected by our segmentation algorithm. The black crosses mark data that have not been processed in Messina et al. (2010). We employed a 50-day sliding window, while in the plot we sketch a 200-day sliding window for clarity.


Fig. 2. Left panel: distribution of detected periods for the star ASAS J070030-7941.8. The black continuous arrow indicates the average rotation period $\left\langle P_{\text {rot }}\right\rangle$. The black dotted arrows show the beat periods. Right panel: period distribution after beat rejections. $P_{\min }$ and $P_{\max }$ are estimated by taking the 5 th and the 95 th percentile of the distribution to ensure that the $\Delta \Omega_{\text {phot }}$ computation is not driven by outliers.

obtained for ASAS J072851-3014.8, ASAS J084229-7903.9, and SWASP1 J101828.70-315002.8, which were taken as representative examples of our targets.

The total number of segments that could be used to measure the rotation period is different for each target. This number is a complex function of the time-series sampling and the stellar rotation period. We divided our data into three quality groups according to the number of segments $N$ in which a period with $F A P<0.01$ is measured. Group A includes stars with $N>200$, group B stars with $N$ between 100 and 200, and group C stars with $N<100$.

\subsection{False-alarm probability estimate}

We computed the false-alarm probability associated with a given period $P$ by using the Schwarzenberg-Czerny (1998) $\beta$ distribution:

$\operatorname{Pr}\left[Z_{\max } \leq z\right]=1-\left(1-(1-2 z)^{(N-3) / 2}\right)^{M}$, 
E. Distefano et al.: Lower limit for differential rotation in members of young loose stellar associations
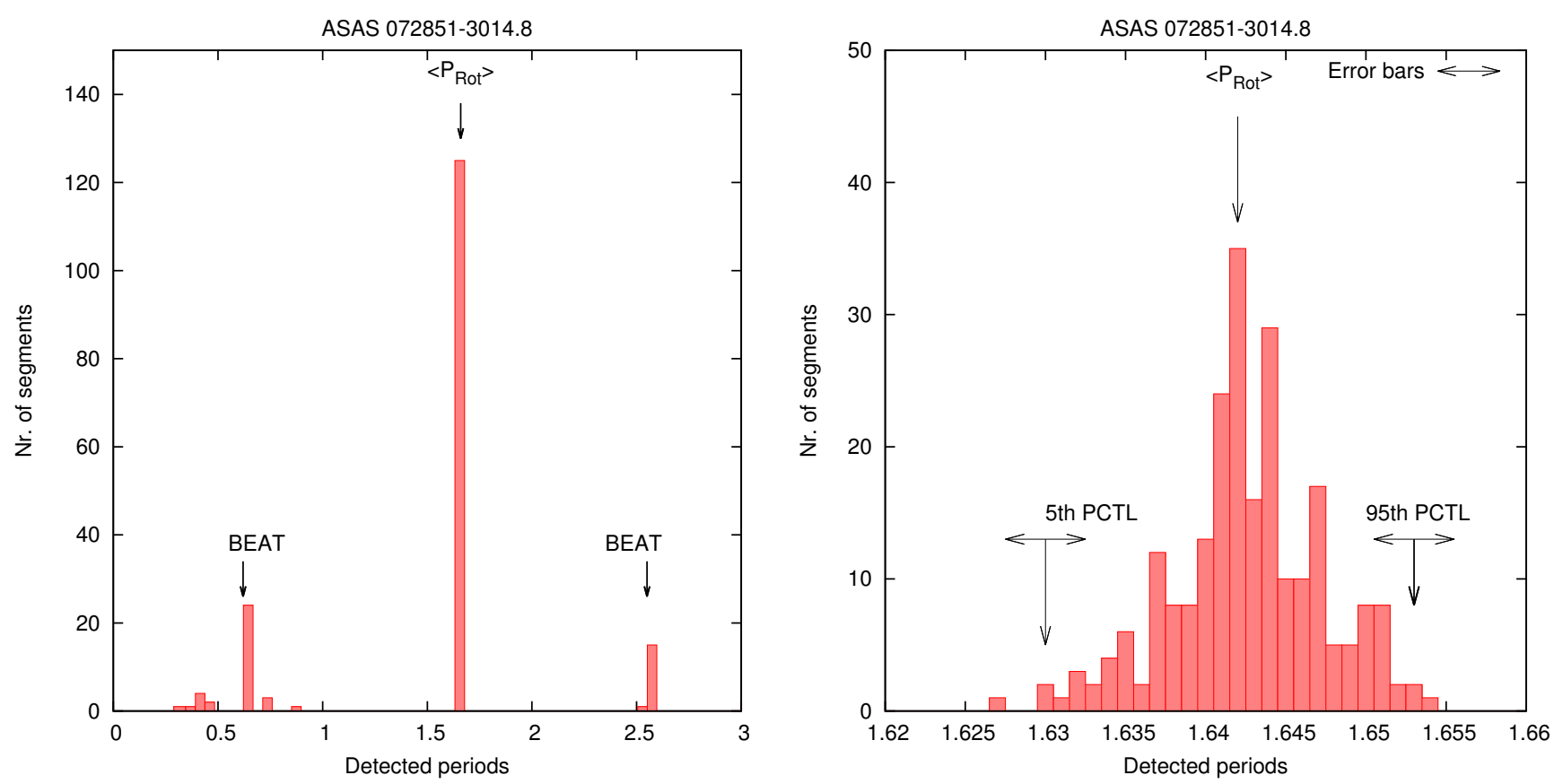

Fig. 3. Same as Fig. 2 for ASAS J072851-3014.8.
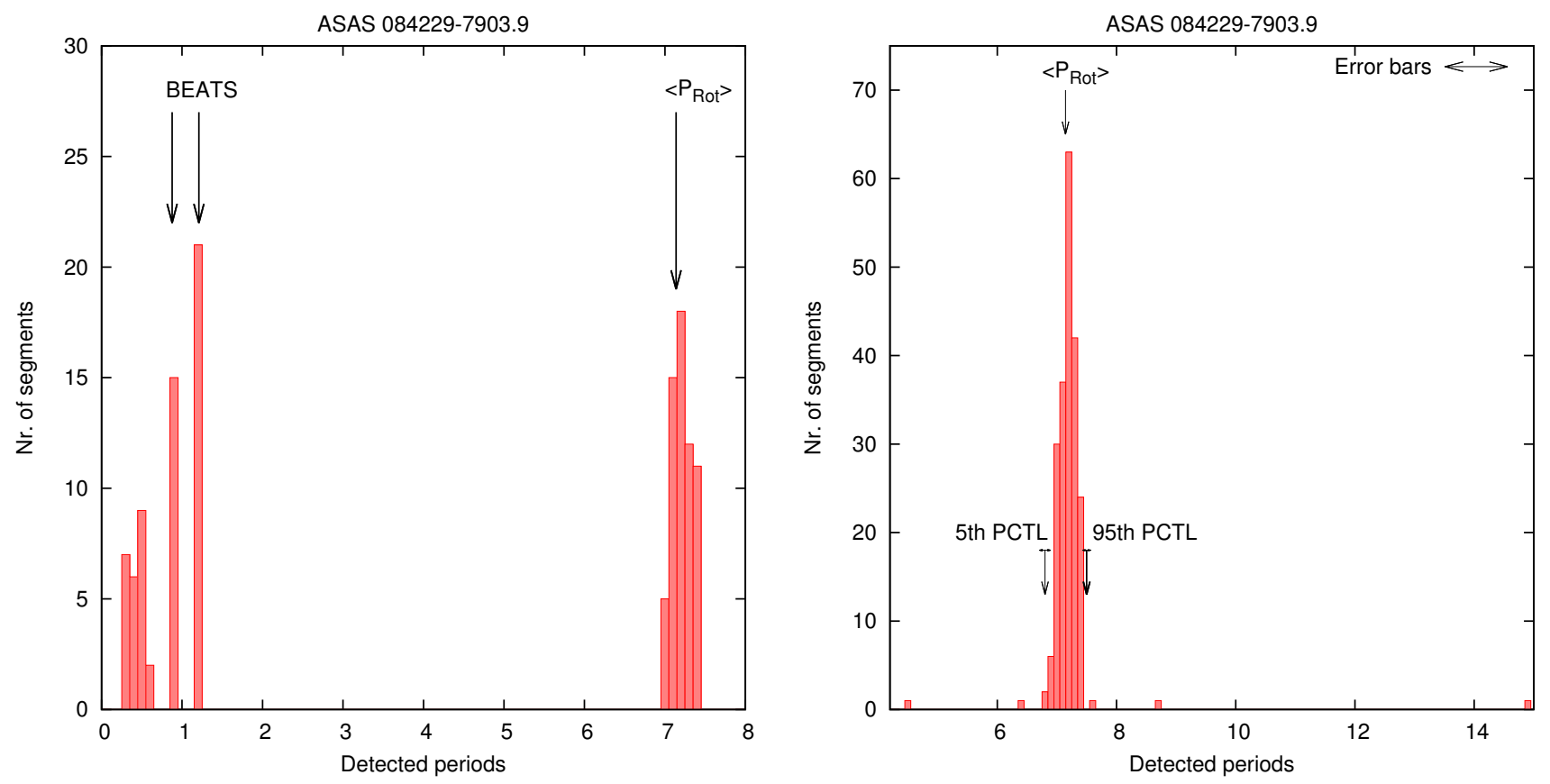

Fig. 4. Same as Fig. 2 for ASAS J084229-7903.0.

where $Z$ is the power related to $P$ in the periodogram, $N$ is the number of data points, and $M$ the number of independent frequencies, that is, the number of frequencies at which the periodogram powers are independent random variables. Unfortunately, for an unevenly sampled time series, $M$ cannot be theoretically derived. We used the value estimated by Distefano et al. (2012),

$M=1.8 N_{\mathrm{f}} / k$,

where $N_{\mathrm{f}}$ is the number of inspected frequencies and $k$ is a factor introduced to take the periodogram oversampling into account. This is defined as $k=(1 / T) / \delta v$, where $T$ is the time interval spanned by the segment and $\delta v=0.0001 \mathrm{~d}^{-1}$ is the frequency

step adopted to sample the periodogram. Equation (3) was derived by Distefano et al. (2012) by fitting empirical distributions of the peak powers $Z$ as generated by Monte Carlo simulations. In our analysis we used only periods with a $F A P \leq 0.01$.

\subsection{Error estimate}

The error associated with $\Delta \Omega_{\text {phot }}$ is given by

$\sigma_{\Delta \Omega_{\text {phot }}}^{2}=\sigma_{\Omega_{\max }}^{2}+\sigma_{\Omega_{\min }}^{2}$,

where $\sigma_{\Omega_{\max }}=2 \pi \sigma_{v_{\max }}$ and $\sigma_{\Omega_{\min }}=2 \pi \sigma_{v_{\min }}$.

The error associated with the frequency detected by the Lomb-Scargle periodogram has two components. One 


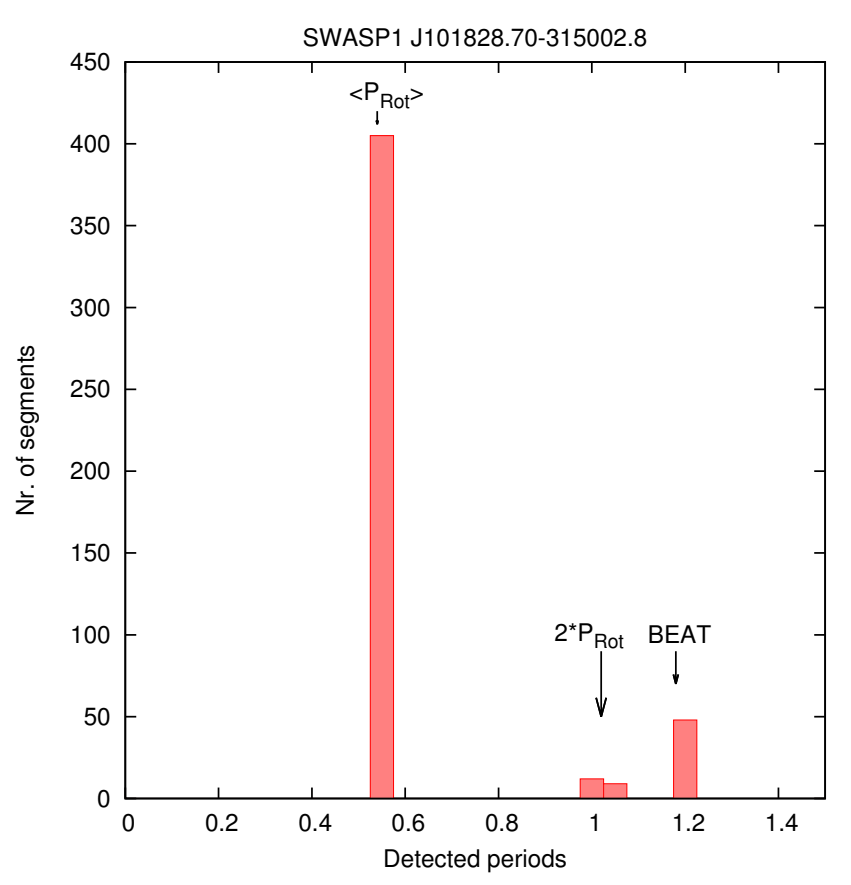

Fig. 5. Same as Fig. 2 for SWASP1 J101828.70-315002.8.

component is due to the limited and discrete sampling of the signal, while the other is due to the data noise. Kovacs (1981) estimated the error $\delta v_{\text {samp }}$ introduced by the sampling for a noisefree sinusoidal signal with frequency $v_{0}$ and found that this error is inversely correlated with $v_{0} T^{2}$, where $T$ is the interval spanned by the time series. Gilliland \& Fisher (1985) showed that the result found by Kovacs (1981) can be approximated by

$\delta v_{\mathrm{samp}}=\frac{0.16}{\sqrt{2} v_{0} T^{2}}$

Kovacs (1981) derived this error for a uniformly sampled signal, but the result can also be applied to unevenly sampled time series. Kovacs (1981) also estimated the error caused by Gaussian white noise as

$\delta v_{\text {noise }}=\frac{3 \sigma}{4 \sqrt{N} v_{0} T A}$,

where $\sigma$ is the standard deviation of the noise, $N$ the number of time-series points, and $A$ the amplitude of the signal. Equation (6) has been derived for white Gaussian noise and can lead to an underestimation of the true error. To estimate the error caused by data noise more realistically, we used the following approach:

- we fit the data with a sinusoid;

- we computed the fit residuals to estimate the data noise;

- we computed 10000 permutations of the residuals and constructed 10000 synthetic time series by adding the permuted residual to the sinusoid;

- we ran the period search algorithm on each synthetic time series; and

- we built the distribution of the detected frequencies and took the standard deviation of this distribution as an error estimate.

The error $\delta v_{\text {samp }}$ increases with the stellar rotation period (see Eq. (5)). It is very small and ranges from $10^{-4} \mathrm{rad} \mathrm{d}^{-1}$ for stars

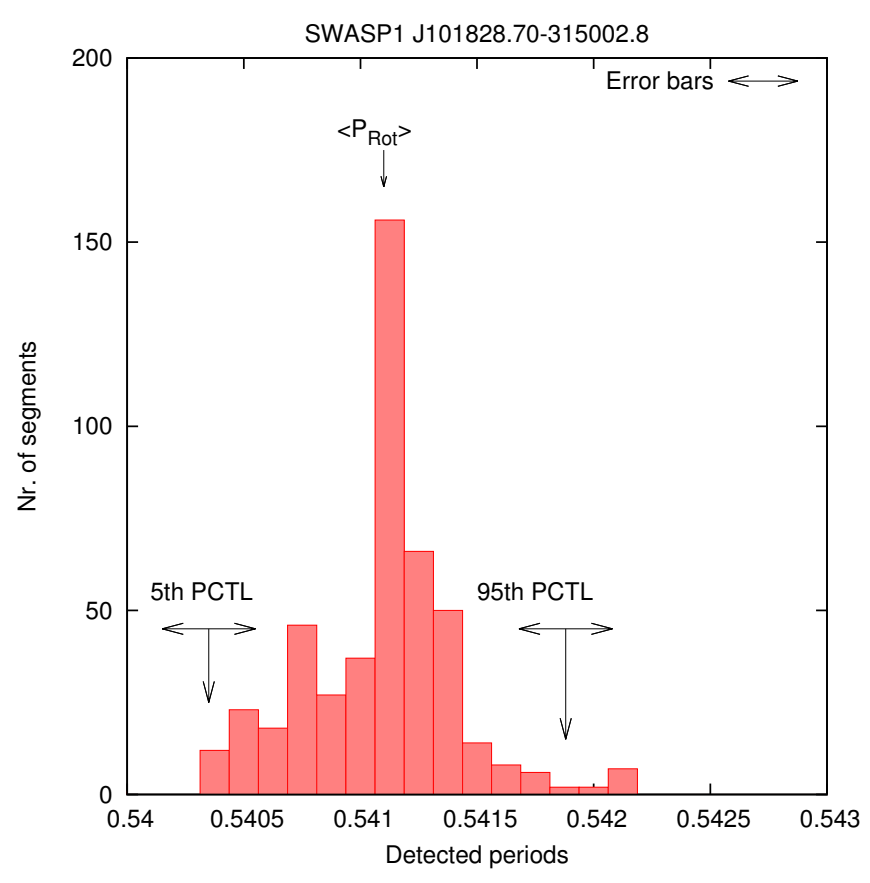

rotating in $0.5 \mathrm{~d}$ to $0.006 \mathrm{rad} \mathrm{d}^{-1}$ for stars rotating in $20 \mathrm{~d}$ (this is the highest rotation period detected in our target stars).

The error $\delta v_{\text {noise }}$ inferred from simulating synthetic time series depends on different factors such as stellar magnitude, variability amplitude, and the number of observations. It ranges between $0.003 \mathrm{rad} \mathrm{d}^{-1}$ and $0.02 \mathrm{rad} \mathrm{d}^{-1}$.

The final errors on $\Delta \Omega_{\text {phot }}$ measurements range between 0.005 and $0.025 \mathrm{rad} \mathrm{d}^{-1}$.

\subsection{Output of the analysis procedure}

In Figs. 6-9 we show the typical output of our analysis for ASAS J070030-7941.8, ASAS J072851-3014.8, ASAS J084229-7903.9, and SWASP1 J101828.70-315002.8

In the top panels we plot the rotation periods detected in the different segments vs. the mid-observation times of the same segments. In the central panels we plot the interquartile range (IQR) of the observed magnitudes, which can be regarded as an index of the variability amplitude, and in the bottom panels we plot the median magnitude. The solid lines were obtained by fitting the data with smoothing cubic splines and are plotted to guide the eye. The period and IQR seem to follow defined patterns well, which suggests that there are cyclical changes in the AR configurations. These cyclical patterns will be investigated in a forthcoming work. In some segments, no significant periods were detected. This can be ascribed to different reasons:

- the amplitude of the rotational modulation is on the same order as the photometric error and therefore is too low to be detected, or

- the AR evolution occurs on a timescale similar to or shorter than the length of the sliding window and therefore masks the variability that is due to rotational modulation.

In Figs. 6-9 we also plot the results obtained by using 100-d and $150-\mathrm{d}$ sliding windows for comparison. In principle, the use of the longer windows should give more precise measurements of the stellar rotation period, as discussed in Sect. 3.4. In fact, the 


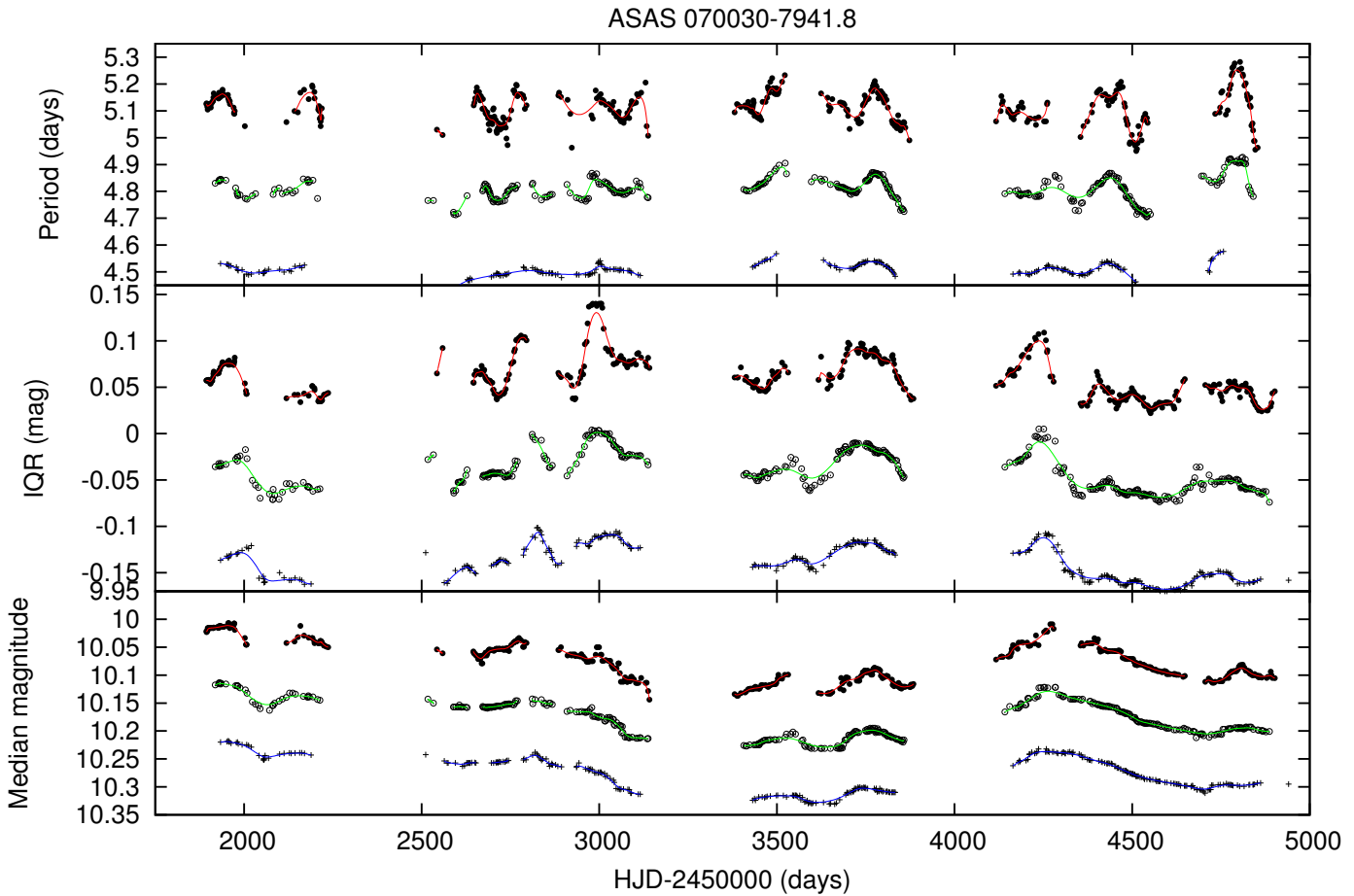

Fig. 6. Output of our analysis for ASAS ID J070030-7941.8. The filled circles, empty circles, and crosses show the results obtained with 50-d, $100-\mathrm{d}$, and 150-d sliding windows, respectively. The results obtained with the 100-d and 150-d sliding window have been vertically shifted to make the plot clearer. Top panel: rotation periods found in the different segments vs. time. Each point is located at the mid-time of the corresponding segment. Middle panel: IQR measured in the different segments vs. time. Bottom panel: median magnitude vs. time. The solid lines were obtained by fitting the data with smoothing cubic splines.

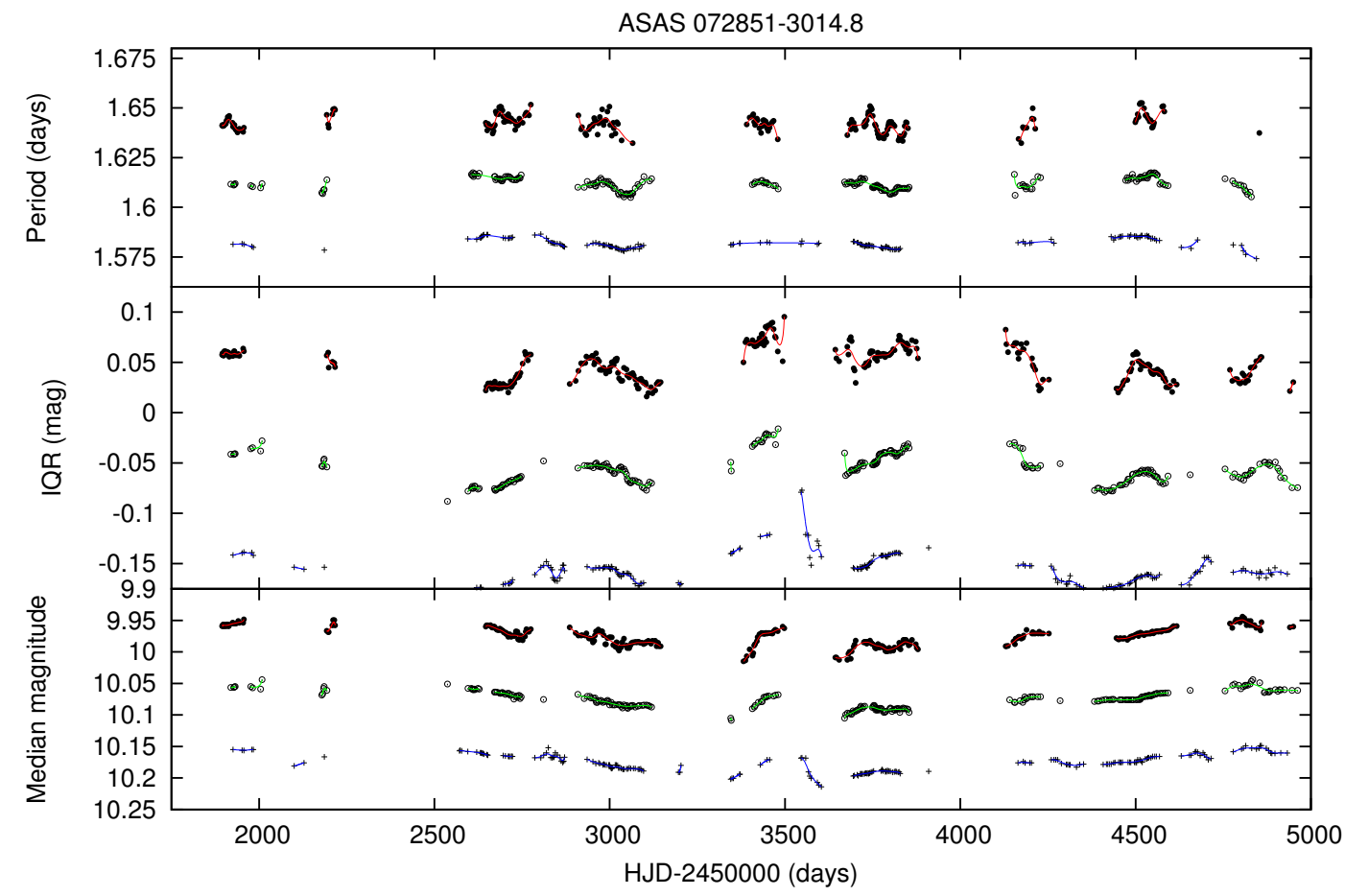

Fig. 7. Same as Fig. 6 for ASAS J072851-3014.8.

equations shown in Sect. 3.4 are strictly valid only for a pure sinusoidal signal. In solar-like variables, the intrinsic evolution of ARs tends to modify the amplitude and phase of the rotational signal, and the use of the longer windows does not improve the precision of the period measurements. Moreover, the use of a given window tends to filter out the variability phenomena occurring on a timescale similar to or shorter than the window length. This produces smoother curves and flattens the amplitude of the period variations. The use of a 50-d window is therefore more suitable to take into account the ARs evolution. Ideally, the length of the window should cover a small number of stellar rotations. Ferreira Lopes et al. (2015), for instance, suggested 


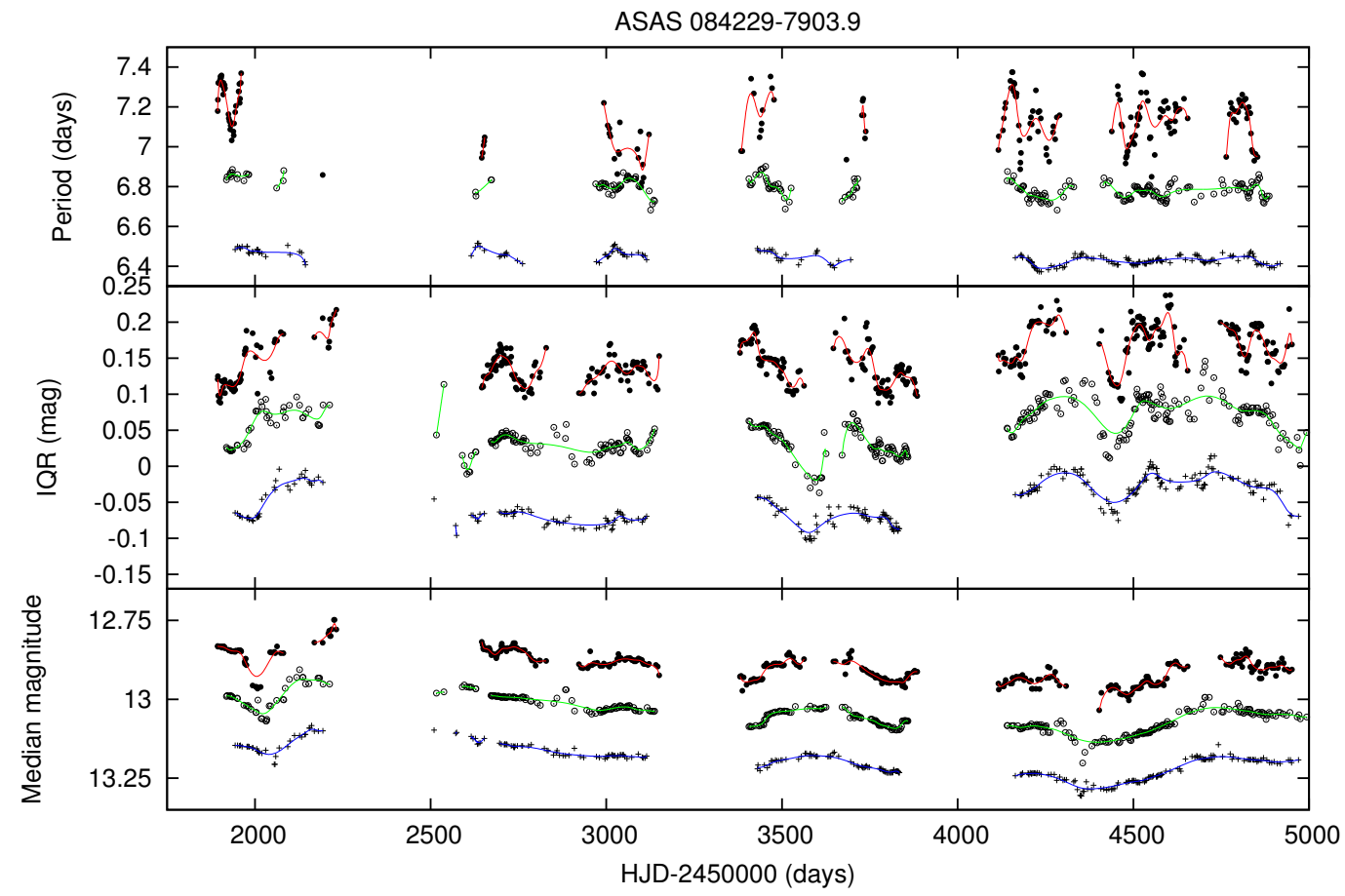

Fig. 8. Same as Fig. 6 for ASAS J0842.29-7903.9.

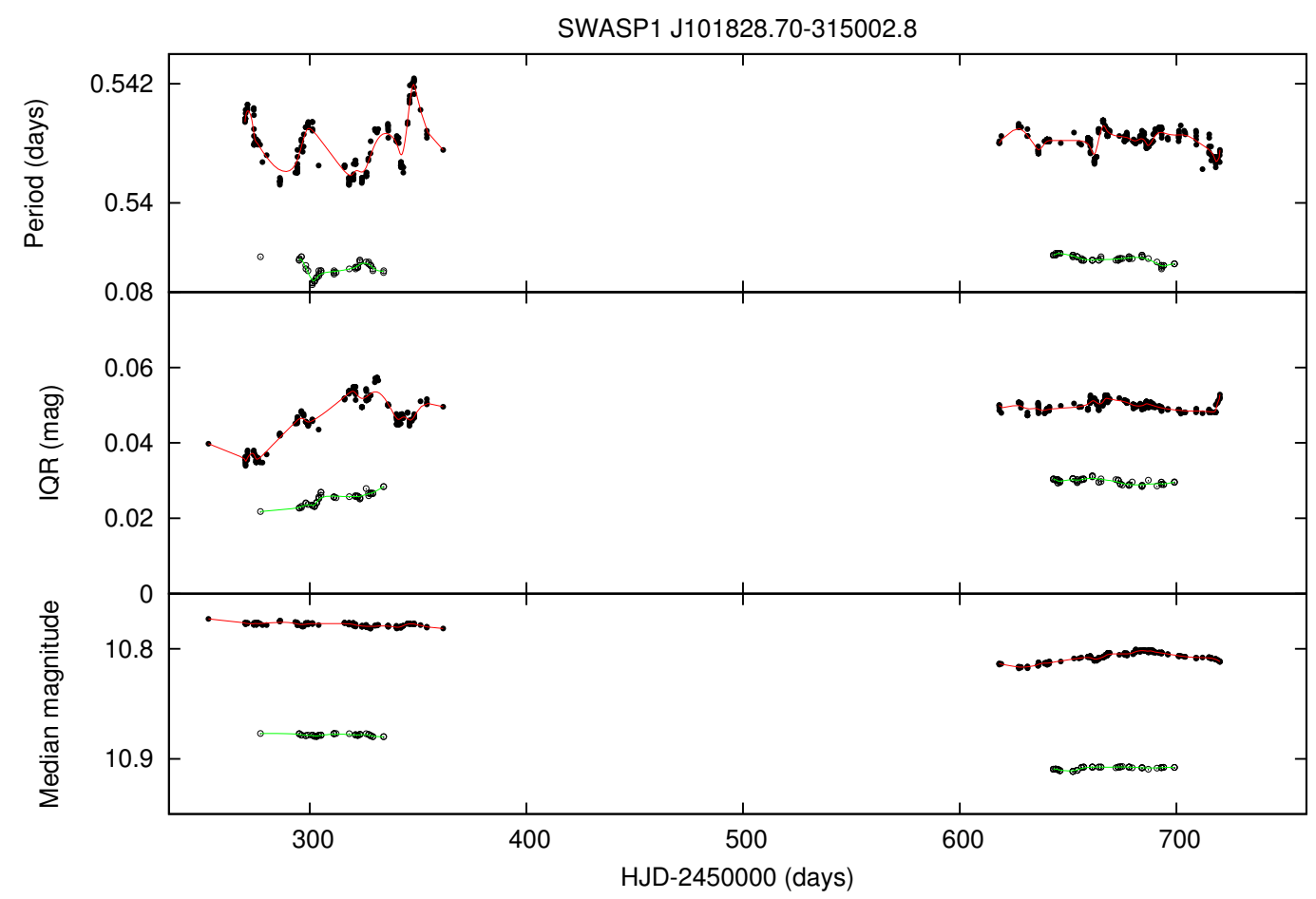

Fig. 9. Same as Fig. 6 for SWASP1 J101828.70-315002.8. The analysis with a 150-d sliding window has not been performed in this case because the two observation season spans are too short.

that long-term time series of solar-like variables should be segmented into sub-series of length $5 \times P_{\text {Rot }}$ to limit the effects of AR evolution. Unfortunately, a similar choice of window length is not feasible for most of our targets because of the sparse timeseries sampling.

\subsection{Comparison with other methods}

In recent years, several methods have been developed to measure the SDR in solar-like stars. These methods can be grouped into two classes. The first class comprises methods based on Doppler imaging (see e.g. Donati et al. 1997; Donati \& Collier Cameron 1997; Strassmeier 2009; Waite et al. 2011), the second comprises methods based on the analysis of photometric data (see, e.g., Donahue et al. 1996; Reinhold et al. 2013; Lanza et al. 2014). Methods based on Doppler imaging are very effective in measuring the SDR in fast-rotating stars $\left(v \sin i \geq 15 \mathrm{~km} \mathrm{~s}^{-1}\right)$ but cannot be successfully applied to slow rotators. Photometric data analyses can be applied to slow rotators, but only allow estimating a lower limit for the SDR. In this section, we discuss the main 
differences between our method and those employed by Waite et al. (2011) and Reinhold et al. (2013), which are taken as representative examples of the two classes.

\subsubsection{Comparison with Zeeman Doppler imaging}

Waite et al. (2011) analyzed spectropolarimetric and photometric data that were acquired during nine nights to study the topology of the magnetic field and the differential rotation of HD 106506. They complemented the information inferred from the Zeeman Doppler imaging (ZDI) with the photometric data and derived an equatorial rotation period $P_{0}=1.39 \pm 0.01 \mathrm{~d}$ and a photospheric shear $\Delta \Omega=0.21 \pm 0.03 \mathrm{rad} \mathrm{d}^{-1}$. They also studied the spot distribution on the stellar surface and found a large polar spot coupled with low- and mid-latitude features. To compare our detection method with that employed by Waite et al. (2011), we applied our analysis to the ASAS time series of HD 106506

The shortest detected period is $P_{\min }=1.39 \mathrm{~d}$, which is equal to the equatorial period found by Waite et al. (2011), while the longest period is $P_{\max }=1.445 \mathrm{~d}$. The shear inferred from $P_{\min }$ and $P_{\max }$ is $\Delta \Omega_{\text {phot }}=0.17 \mathrm{rad} \mathrm{d}^{-1}$, that is, about 20 per cent lower than the value found by Waite et al. (2011). This difference is expected because the photometric analysis fails to detect the stellar rotation frequency at high latitudes: polar spots are always visible and do not induce an appreciable flux modulation.

\subsubsection{Comparison with short-term photometry analysis}

Reinhold et al. (2013) measured a lower limit of the SDR for about 20000 late-type stars by analyzing the Q3 long-cadence photometric time-series collected by the Kepler mission. These time-series cover an interval of about $90 \mathrm{~d}$ and therefore are not suitable for segmentation. Reinhold et al. (2013) computed the Lomb-Scargle periodogram for the whole $90 \mathrm{~d}$ time series and estimated the amplitude of the SDR as

$\Delta \Omega_{\text {phot }}=\Omega_{1}-\Omega_{2}$,

where $\Omega_{1}=\frac{2 \pi}{P_{1}}, \Omega_{2}=\frac{2 \pi}{P_{2}}$, and $P_{1}$ and $P_{2}$ are the two most highly significant periods detected in the periodogram. Equation (7) is based on the assumption that $P_{1}$ and $P_{2}$ are the rotation periods of two AR complexes located at different latitudes $\theta_{1}$ and $\theta_{2}$. This way of measuring has two drawbacks:

- The latitude range covered by $\theta_{1}$ and $\theta_{2}$ depends on the phase of the stellar magnetic cycle at which the time series was collected. If a star has a solar-like cycle, the ARs gradually migrate from high latitudes (at the minimum of cycle) to the equator (at the maximum).

- It is based on the detection of multiperiodicities in the same time series and assumes that the secondary period $P_{2}$ is due to rotational modulation. However, as Aigrain et al. (2015) pointed out, the secondary peak in the Lomb-Scargle periodogram might also be induced by an intrinsic evolution of the ARs.

The method employed here has two advantages over the technique used by Reinhold et al. (2013). First of all, it is based on the analysis of long-term photometry. The use of ASAS and Super-WASP time series permits us to study our target stars over a timescale comparable with the activity cycle duration and to track stellar rotation in a wider range of latitudes. This reduces the measurement bias that is due to the use of short-term photometry. The second advantage is that our method is not based on multiple periodicities, but searches for a drift in the primary period over the whole time series. Aigrain et al. (2015) tested different measurement methods on simulated light curves and showed that the use of multiple peaks can sometimes fail in detecting and measuring the SDR. The same authors point out that a more reliable SDR estimate can be achieved by searching for a drift in the mean period over an activity cycle.

\section{Results}

In Table 3 we report our $\Delta \Omega_{\text {phot }}$ measurements. If for a given source both ASAS and SuperWASP estimates are available, the highest $\Delta \Omega_{\text {phot }}$ value is reported. In the last column of the table we indicate whether the photometric shear was computed with ASAS or SuperWASP data.

\subsection{Correlation between SDR and global stellar parameters}

We studied the relation of our $\Delta \Omega_{\text {phot }}$ measurements to the astrophysical parameters of our targets. The astrophysical parameters were inferred by using the infrared magnitudes $M_{J}$ and $M_{H}$ and the theoretical isochrones developed by Baraffe et al. (1998), Siess et al. (2000), and Spada et al. (2013). We used infrared magnitudes because the flux contrast between the spots and photosphere is lower in these wavelength passbands. Hence these magnitudes are less affected by the variability induced by rotational modulation in comparison with the optical magnitudes. For a given star, $M_{J}$ and $M_{H}$ are computed by adding the distance modulus $D M$ to the $J$ and $H$ magnitudes reported in the 2MASS catalog. The distance modulus is computed by using the stellar parallax or the distance reported in the literature. We did not apply any correction for reddening because the difference between the observed colors and those expected from the spectral type is within the photometric errors of the 2MASS photometry. The expected colors were taken from the list compiled by Pecaut \& Mamajek (2013) for the pre-main-sequence stars of different spectral types. In Table 4 we report the distance moduli and the inferred $M_{J}$ and $M_{H}$ magnitudes. In Table 5 we report for each star the mass and effective temperature inferred from the comparison with the different models. We also report the global convective turnover timescale $\tau_{\mathrm{C}}$ inferred from the models of Spada et al. (2013) and the derived Rossby number $R=\frac{P_{\text {rot }}}{\tau_{\mathrm{C}}}$. The global convective turnover timescale represents the characteristic time for the rise of a convective element through the stellar convection zone (see Appendix A of Spada et al. 2013, for details on its computation).

\subsubsection{Correlation between the SDR and stellar temperature}

In Fig. 10 we plot $\Delta \Omega_{\text {phot }}$ vs. the color index $B-V$ of our targets. Different symbols are used to mark stars belonging to different associations. Although the data are quite scattered, we note a general trend with SDR amplitude increasing toward bluest colors. The scatter can be ascribed to two different reasons. First of all, it is related to the intrinsic limitations of our measurement method that allows only the detection of a lower limit for the SDR (see discussion in Sect. 3). The second reason is that the plot mixes stars with different ages and rotation periods. Despite the scatter, the trend shown in the picture agrees with the theoretical models developed by Kitchatinov \& Rüdiger (1999) and Küker \& Rüdiger (2011). In these models, the amplitude of stellar SDR is inversely correlated with the depth of the convective zone, and it therefore increases toward higher temperatures. 

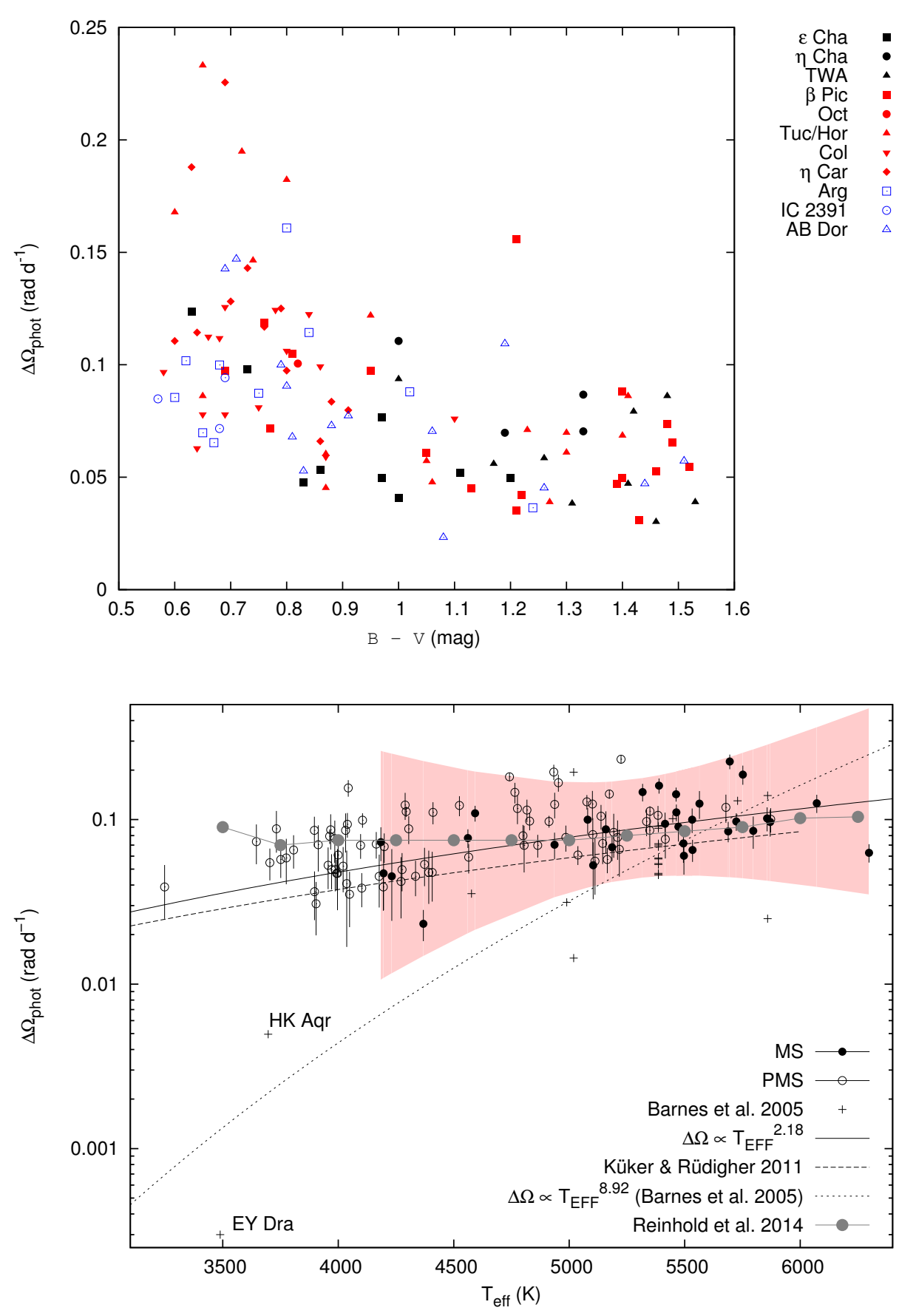

Fig. 10. Quantity $\Delta \Omega_{\text {phot }}$ measured in the present work vs. the color index $B-V . \Delta \Omega_{\text {phot }}$ increases toward the bluest stars. The different symbols are used to mark stars belonging to different associations.

Fig. 11. $\Delta \Omega_{\text {phot }}$ vs. the effective temperature $T_{\text {eff }}$. The black bullets indicate the values measured in the present work. The filled bullets show stars that have reached the ZAMS, and the empty bullets show stars that are still contracting. The black crosses indicate the data reported in Barnes et al. (2005). The continuous and the dotted line depict the power law inferred in the present work and that inferred in Barnes et al. (2005). The dashed line indicates the power law predicted by the theoretical model of Küker \& Rüdiger (2011). The $T_{\text {eff }}$ values have been inferred from the models of Siess et al. (2000). The shaded area represents the uncertainty in our power-law fitting. The large gray bullets and the gray line represent the results of Reinhold et al. (2013).
To quantitatively compare our results and those predicted by Küker \& Rüdiger (2011), we studied the correlation between $\Delta \Omega_{\text {phot }}$ and the stellar temperature.

In Fig. 11 we plot $\Delta \Omega_{\text {phot }}$ vs. the effective temperature $T_{\text {eff }}$ inferred from the Siess et al. (2000) isochrones. The model of Küker \& Rüdiger (2011) predicts the relation $\Delta \Omega=$ $0.071\left(\frac{T_{\text {eff }}}{5500}\right)^{2}$ for ZAMS stars with $T_{\text {eff }} \leq 6000 \mathrm{~K}$. Our targets comprise post-main sequence (PMS) stars that are still contracting and stars recently settled on the main sequence (MS). We selected MS stars by comparing the masses and ages of our targets with the values tabulated by Siess et al. (2000) for the ZAMS stars. We fit our ZAMS data with a power law and found $\Delta \Omega_{\text {phot }}=0.09\left(\frac{T_{\text {eff }}}{5500}\right)^{2.18 \pm 0.65}$.

The use of Baraffe et al. (1998) or Spada et al. (2013) isochrones does not change this result significantly. The power laws obtained with the two models are $\Delta \Omega_{\text {phot }}=$ $0.105\left(\frac{T_{\text {eff }}}{5500}\right)^{2.06 \pm 0.7}$ and $\Delta \Omega_{\text {phot }}=0.09\left(\frac{T_{\text {eff }}}{5500}\right)^{2.6 \pm 0.6}$, respectively. Although the data are quite scattered, the exponents of the theoretical and fitted power laws agree well with each other. However, the two curves exhibit a small offset of about $0.01 \mathrm{rad} \mathrm{d}^{-1}$ and our measurements are, on average, higher than the values predicted by Küker \& Rüdiger (2011). We recall that our analysis tends to underestimate the SDR, hence the shift between theoretical and real values might be more pronounced than that shown in the figure. In Fig. 11 we also plot the results of Reinhold et al. (2013) and those from Barnes et al. (2005) for comparison. We plot the median $\Delta \Omega_{\text {phot }}$ values found by Reinhold et al. (2013) in different $T_{\text {eff }}$ bins. These median values fall inside the $95 \%$ confidence region of our fitted power law. Hence the two works can be considered to agree. We note, 


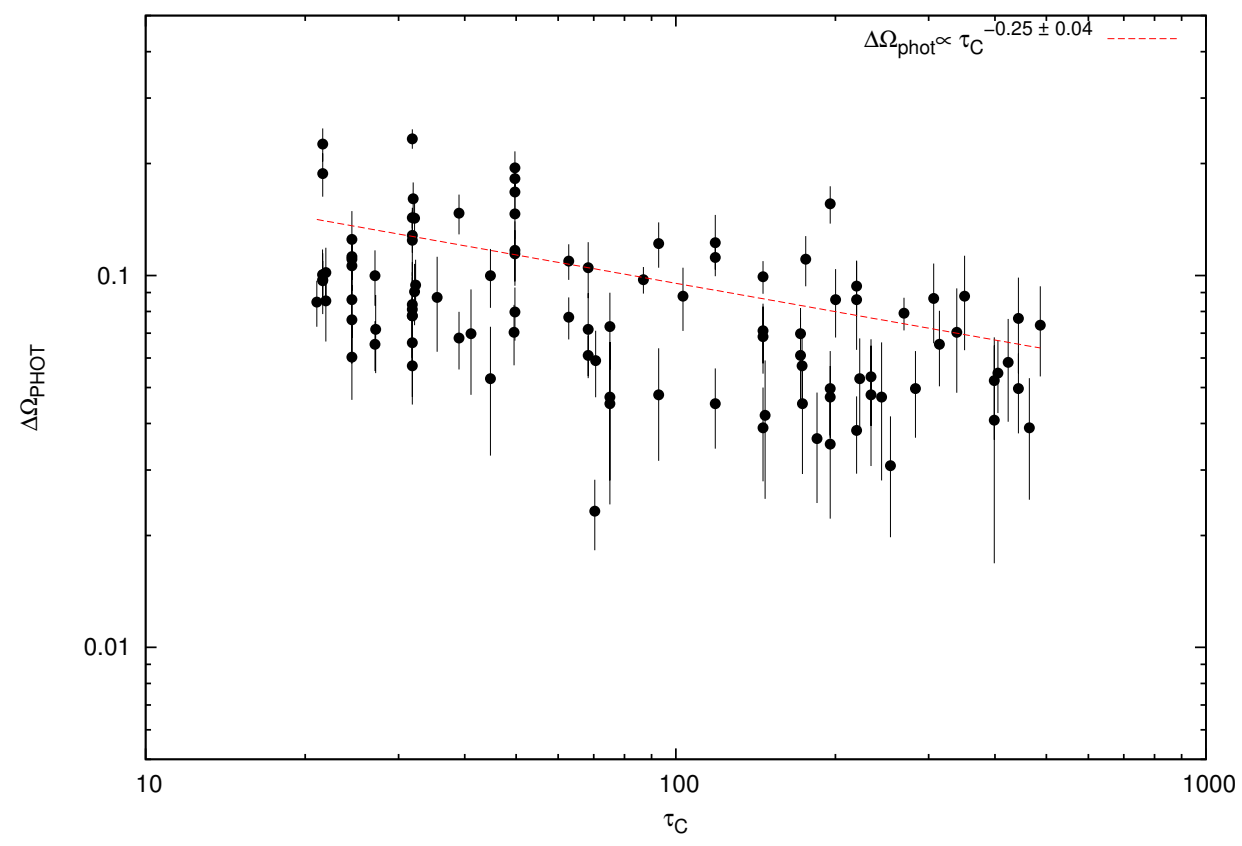

Fig. 12. $\Delta \Omega_{\text {phot }}$ vs. the convective turnover timescale $\tau_{\mathrm{C}}$. however, that the trend found by Reinhold et al. (2013) is flatter than the power law found here and than that predicted by Küker \& Rüdiger (2011). This discrepancy may arise because Reinhold et al. (2013) mixed stars with different ages and with a wider range of rotation periods.

Barnes et al. (2005) analyzed a sample of ten young late-type stars and found the relation $\Delta \Omega \propto T_{\mathrm{eff}}^{8.92}$. The targets investigated by Barnes et al. (2005) comprise PMS stars and stars recently settled on the ZAMS. Their power law is much steeper than that predicted by Küker \& Rüdiger (2011) and disagrees with the trend of our PMS and ZAMS data. Hence our targets do not confirm the finding of Barnes et al. (2005).

\subsubsection{Correlation between the SDR and the global convective turnover timescale}

The stars we analyzed span the age range 4-95 Myr. During this time interval, the stellar structure evolves from a fully convective structure to a radiative core plus a convective envelope. In our sample, stars with the same effective temperature can therefore have very different structures depending on their age and mass. In this age range, the convective turnover timescale $\tau_{\mathrm{C}}$ may be a more convenient parameter for investigating the relation between the SDR and the stellar structure. Indeed, $\tau_{\mathrm{C}}$ is proportional to the depth of the convective zone and is more representative of the stellar structure than of the effective temperature.

In Fig. 12 we plot $\Delta \Omega_{\text {phot }}$ vs. $\tau_{\mathrm{C}} . \Delta \Omega_{\text {phot }}$ increases toward lower $\tau_{\mathrm{C}}$ values. This means that $\Delta \Omega_{\text {phot }}$ increases with decreasing depth of the convective envelope. This result agrees with the theoretical models of Kitchatinov \& Rüdiger (1999), Küker \& Rüdiger (2011), and Küker et al. (2011). We fit a power law to our data and found $\Delta \Omega_{\text {phot }} \propto \tau_{\mathrm{C}}^{-0.25 \pm 0.04}$ (red line). Although the data exhibit a clear trend, they are broadly spread around the fitted power law. This spread is partly caused by the limitations of our measurement method and partly by the mix of stars with different rotation periods in the plot.

\subsubsection{Correlation between the SDR and the rotation period}

In the top panel of Fig. 13 we plot $\Delta \Omega_{\text {phot }}$ vs. the rotation period $P_{\text {rot }}$. In this case, the data do not follow a well-defined trend and cannot be reproduced by a power law. However, the SDR seems to be related to the stellar rotation period. The highest values of $\Delta \Omega_{\text {phot }}$ correspond to $P_{\text {rot }}$ between 0.7 and 5 d. Küker $\&$ Rüdiger (2011) studied with great detail the dependence of the SDR on the rotation period in ZAMS stars. They computed differential rotation for stars with different masses and periods and derived a set of rotational tracks. Each track shows the dependence of $\Delta \Omega$ on the rotation period for a fixed stellar mass. In the bottom panel of Fig. 13 we plot the rotational tracks derived by Küker \& Rüdiger (2011) for stars with 0.5, 0.7, 0.9, and $1.1 M_{\odot}$. According to these tracks, the SDR is almost independent of the stellar rotation period and is more influenced by the stellar mass. We overplot our results on the tracks for comparison. We report only stars that have reached the ZAMS because the tracks were computed for ZAMS stars. The masses of these targets cover the range $0.6-1.35 M_{\odot}$. The circles size is proportional to the stellar mass. Our results show a trend that partly resembles the rotational tracks. The less massive stars exhibit on average a lower SDR. However, stars with $P_{\text {rot }}$ between 0.7 and $2 \mathrm{~d}$ diverge strongly from the model predictions. This suggests that the stellar mass cannot be the main parameter on which the SDR depends, as claimed by Küker \& Rüdiger (2011), and that stellar rotation period plays a key role as well.

\subsubsection{Correlation between the SDR and stellar age}

The stellar associations we studied span an age range between 4 and 95 Myr. Hence our sample includes stars that are in the first phases of the PMS stage and stars that are approaching or have recently settled on the MS. Our results are therefore useful to investigate how the SDR evolves in time during the PMS stage. In the top panel of Fig. 14 we display the measured values of $\Delta \Omega_{\text {phot }}$ vs. the stellar age. The median $\Delta \Omega_{\text {phot }}$ increases between 4 and $30 \mathrm{Myr}$ and then decreases and approaches the current solar value at $95 \mathrm{Myr}$. The scatter of the data is partly caused by the intrinsic limitations related to our methodology and partly by the mixed stellar masses and rotation periods. In the bottom panel of Fig. 14 we report only stars with masses between 0.85 and $1.15 M_{\odot}$ and investigate how $\Delta \Omega_{\text {phot }}$ evolves in a young Sun. The median $\Delta \Omega_{\text {phot }}$ is constant in the first $17 \mathrm{Myr}$, then it significantly 

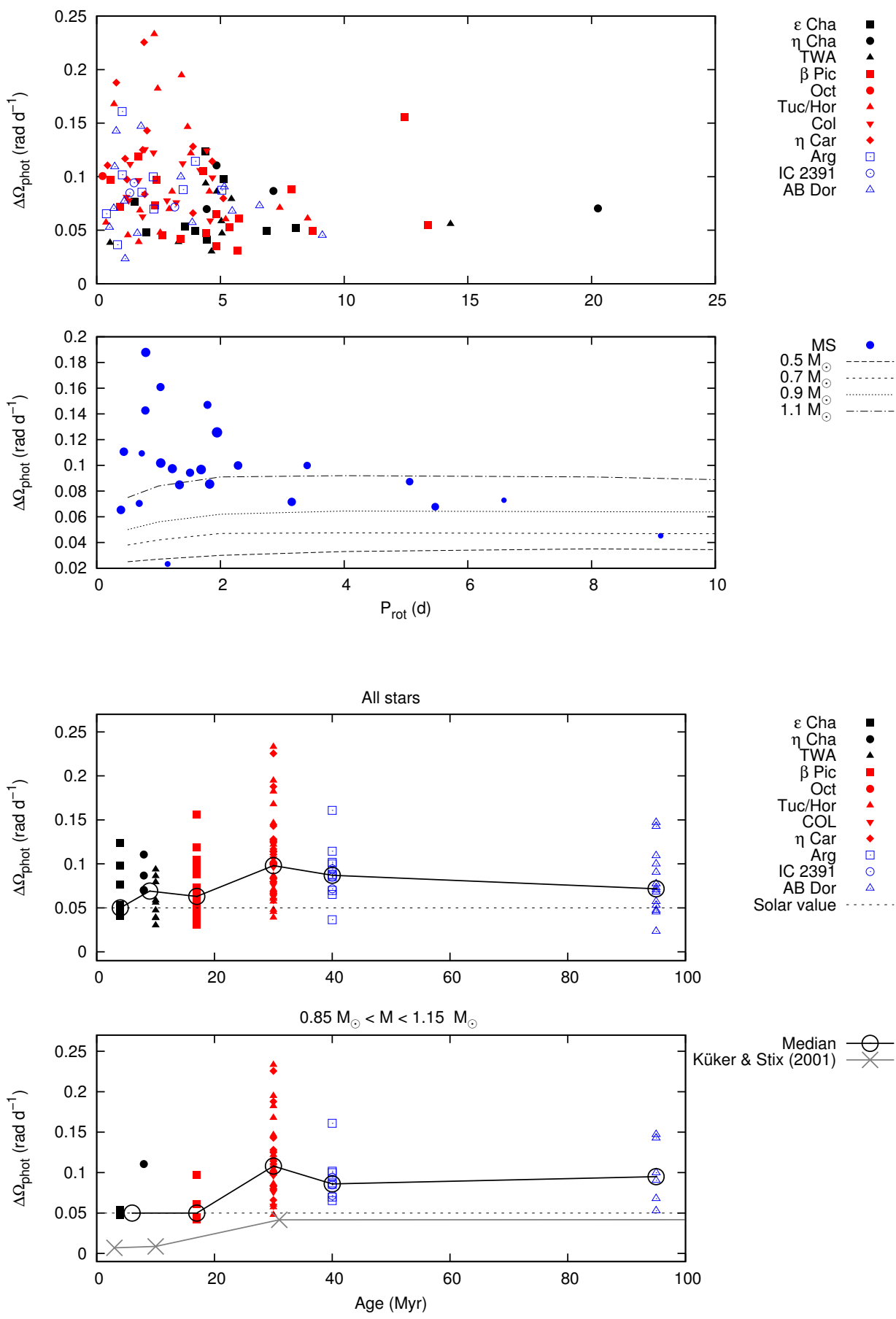

Fig. 13. Top panel: $\Delta \Omega_{\text {phot }}$ vs. the stellar rotation period $P_{\text {rot }}$. The highest $\Delta \Omega_{\text {phot }}$ values have been measured in stars with $P_{\text {rot }} \leq$ 5d. Bottom panel: the same plot restricted to the MS stars. The symbol sizes are proportional to the stellar masses, which range between 0.6 and $1.35 M_{\odot}$. The black lines are the rotational tracks derived by Küker \& Rüdiger (2011) for stars of 0.5, 0.7, 0.9, and $1.1 M_{\odot}$ as labeled.
Fig. 14. Top panel: $\Delta \Omega_{\text {phot }}$ vs. stellar age. The empty circles indicate the median $\Delta \Omega_{\text {phot }}$ values measured at different ages (note that the members of $\eta C h a$ have been grouped with the members of TWA). The black continuous line connects the median $\Delta \Omega_{\text {phot }}$ values and the dotted line marks the present solar shear for comparison. Bottom panel: the same plot restricted to stars with mass between 0.9 and $1.1 M_{\odot}$. The gray crosses mark the rotational shear for the Sun at different ages, as predicted by Küker \& Stix (2001). increases, and at $30 \mathrm{Myr}$, its value is about twice the initial value. Between 30 and 95 Myr $\Delta \Omega_{\text {phot }}$ remains almost constant. Küker $\&$ Stix (2001) modeled the SDR evolution in the Sun. They developed four theoretical models for the Sun at 3,10, and $31 \mathrm{Myr}$ and for the present Sun. In their models $\Delta \Omega$ is inversely correlated to the depth of the convective zone. This implies that $\Delta \Omega$ increases between 3 and 31 Myr. At $3 \mathrm{Myr}$, the Sun has a fully convective structure and a low SDR. As the Sun evolves in time, a radiative core grows, the depth of the convective zone decreases, and $\Delta \Omega$ increases. Küker \& Stix (2001) did not find a significant difference between the $31 \mathrm{Myr}$ and the present Sun models. Hence, according to their work, the Sun should have reached its current $\Delta \Omega$ value at about $30 \mathrm{Myr}$. In this case, our results also agree only qualitatively with the model prediction. The median $\Delta \Omega_{\text {phot }}$ values increase between 4 and $30 \mathrm{Myr}$, but they are systematically higher than those predicted by Küker \& Stix (2001). We note that although these young stars exhibit an SDR greater than solar, they can be regarded as solid-body rotators because they rotate faster than the present Sun and have a low relative shear $\alpha=\frac{\Delta \Omega}{\Omega_{\mathrm{eq}}}$.

\section{Discussion}

In the previous section we showed that our results agree qualitatively with the models developed by Kitchatinov \& Rüdiger (1999) and Küker \& Rüdiger (2011). However, our $\Delta \Omega_{\text {phot }}$ measurements are often higher than the values predicted by the same models (see Figs. 11 and 13), especially in stars with periods between 0.7 and $2 \mathrm{~d}$. This disagreement between $\Delta \Omega$ values and model predictions has previously been noted and discussed by 

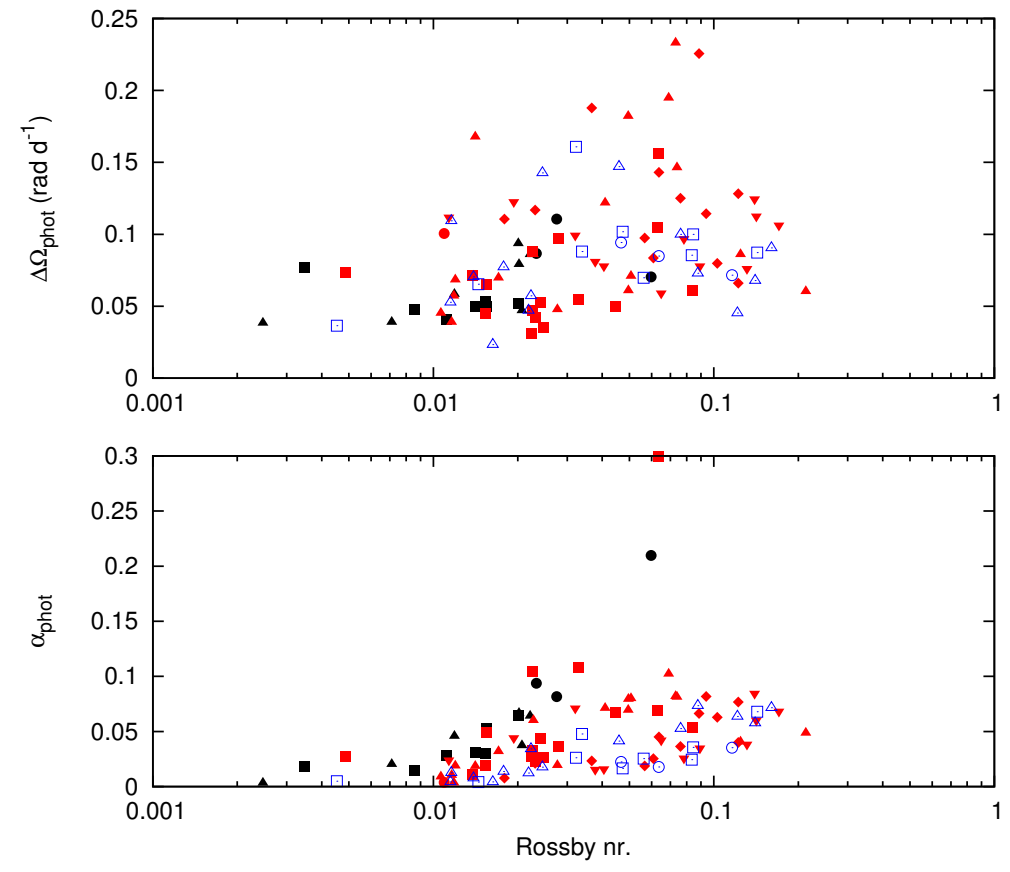

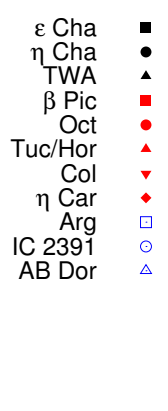

Fig. 15. Top panel: absolute shear $\Delta \Omega$ vs. the Rossby number Ro. Bottom panel: the relative shear $\alpha$ vs. Ro. $\Delta \Omega$ and $\alpha$ increase toward higher $R o$ values. This trend agrees with the model developed by Gastine et al. (2014).
Marsden et al. (2006). These authors measured an absolute shear $\Delta \Omega=0.4 \mathrm{rad} \mathrm{d}^{-1}$ for the $G 0$ star HD 171488. This value is significantly higher than those measured for the $G$ dwarfs R58 and LQ Lup, which are $\Delta \Omega=0.138 \mathrm{rad} \mathrm{d}^{-1}$ and $\Delta \Omega=0.12 \mathrm{rad} \mathrm{d}^{-1}$, respectively (Marsden et al. 2005; Donati et al. 2000). According to the models, the three stars should have about the same rotational shear because the stellar temperature is the main parameter on which $\Delta \Omega$ depends. Küker et al. (2011) were unable to explain this discrepancy and questioned the reliability of the $\Delta \Omega$ measurement for HD 171488. However, Jeffers \& Donati (2008) made an independent measurement and found $\Delta \Omega=0.5 \mathrm{rad} \mathrm{d}^{-1}$ The main difference between HD 171488 and the other two stars lies in the rotation period. HD 171488 has a rotation period of $1.31 \mathrm{~d}$, while the other two stars have a rotation period of 0.5 and $0.3 \mathrm{~d}$, respectively.

The rotational tracks computed by Küker \& Rüdiger (2011) predict that the SDR amplitude slightly increases with the period in the range $0.3-2 \mathrm{~d}$ (see bottom panel of Fig. 13), but this increment is too small to explain the SDR amplitude of HD 171488. The high differential rotation found in HD 171488 and in our targets, with periods between 0.7 and $2 \mathrm{~d}$, suggests that the dependence on the rotation period could be more pronounced than models prediction.

A stronger dependence on the stellar rotation rate could also explain the discrepancy between the power law found by Barnes et al. (2005) and that found in the present work (see Fig. 11). The high steepness of the power law found by Barnes et al. (2005) is due to the M1V stars HK Aqr and EY Dra, for which the authors measured very low SDR values of 0.005 and $0.0003 \mathrm{rad} \mathrm{d}^{-1}$, respectively. The M1V stars of our sample have higher $\Delta \Omega_{\text {phot }}$ values. However, we point out that HK Aqr and EY Dra rotate in about $0.5 \mathrm{~d}$, while the M1V stars investigated here have rotation periods of between 1.5 and $5.5 \mathrm{~d}$. The difference between HK Aqr, EY Dra, and the M1V stars investigated here might therefore be due to the different rotation periods.

The models developed by Küker \& Rüdiger (2011) are based on the so-called $\Lambda$ effect. In these models the main driver of the differential rotation is the non-diffusive term of the Reynolds stress induced by the interaction between the Coriolis force and the convective motions. As noted by Gastine et al. (2014), these models depend on free parameters such as the turbulent viscosity coefficients. A different choice of these parameters might lead to a stronger dependence on stellar rotation rate. Gastine et al. (2014) developed a model based on a 3D hydrodynamical code where the Reynolds stresses do not need to be parametrized. In this model, the main parameter on which $\Delta \Omega$ depends is the Rossby number $R o=\frac{P_{\text {rot }}}{\tau_{\mathrm{c}}}$, where $P_{\text {rot }}$ is the stellar rotation period and $\tau_{\mathrm{c}}$ is the convective turnover timescale. If $R o<1$, which is the case of our targets, the Coriolis force dominates the buoyancy and the star tends to rotate as a solid body. As Ro increases, the Coriolis force becomes weaker and the value of differential rotation increases. For $R o>1$ the buoyancy dominates on Coriolis force and the SDR becomes antisolar. In Fig. 15 we plot $\Delta \Omega_{\text {phot }}$ vs. the Rossby number computed in Sect. 3. The plot is quite scattered, but the trend of $\Delta \Omega_{\text {phot }}$ seems to confirm the prediction of Gastine et al. (2014). In the bottom panel of Fig. 15 we report the relative shear $\alpha_{\text {phot }}$ vs. the Rossby number. The trend and the range of $\alpha_{\text {phot }}$ values are very similar to that predicted by Gastine et al. (2014), which is shown in their Fig. 2.

\section{Conclusions}

We investigated the correlation between the SDR amplitude and global stellar parameters in members of young loose stellar associations. We measured the quantities $\Delta \Omega_{\text {phot }}$ and $\alpha_{\text {phot }}$ for 111 stars by processing long-term photometric time-series. These quantities are lower limits to the absolute and to the relative surface rotational shear. Our analysis led to the following results:

- $\Delta \Omega_{\text {phot }}$ increases with effective temperature $T_{\text {eff }}$ following the power law $\Delta \Omega_{\text {phot }} \propto T_{\text {eff }}^{2.18 \pm 0.65}$ in MS stars. This power law is very close to that predicted by Küker $\&$ Rüdiger (2011; i.e., $\Delta \Omega \propto T_{\text {eff }}^{2}$ ).

- The PMS stars of our sample show a trend very similar to that exhibited by MS stars. Thus, the power law $\Delta \Omega \propto T_{\text {eff }}^{8.6}$ found by Barnes et al. (2005) for PMS and ZAMS stars is not confirmed by our measurements. 
- $\Delta \Omega_{\text {phot }}$ increases with decreasing convective turnover timescale $\tau_{\mathrm{C}}$ according to the power law $\Delta \Omega_{\text {phot }} \propto \tau_{\mathrm{C}}^{-0.25 \pm 0.4}$.

- Our $\Delta \Omega_{\text {phot }}$ measurements are systematically higher than the values predicted by Küker \& Rüdiger (2011). This discrepancy is particularly large in stars with a rotation period of between 0.7 and $2 \mathrm{~d}$ and suggests that the dependence on the rotation period may be stronger than the model prediction.

- We investigated the time evolution of $\Delta \Omega_{\text {phot }}$ for a $1 M_{\odot}$ stars and found that $\Delta \Omega_{\text {phot }}$ increases with stellar age in the first 30 Myr. This is consistent with the theoretical models that predict a low degree of differential rotation for fully convective stars.

- $\Delta \Omega_{\text {phot }}$ and $\alpha_{\text {phot }}$ increase with the Rossby number $R o$ in agreement with the theoretical model developed by Gastine et al. (2014).

Acknowledgements. The extensive use of the SIMBAD and ADS databases operated by the CDS centre, Strasbourg, France, is gratefully acknowledged. We used data from the WASP public archive in this research. The WASP consortium comprises of the University of Cambridge, Keele University, University of Leicester, The Open University, The Queen's University Belfast, St. Andrews University, and the Isaac Newton Group. Funding for WASP comes from the consortium universities and from the UK's Science and Technology Facilities Council. The Authors would like to thank Dr. G. Pojmanski for the extensive use we made of the ASAS database. We are grateful to Rainer Arlt and to the anonymous referee for helpful comments and suggestions.

\section{References}

Aigrain, S., Llama, J., Ceillier, T., et al. 2015, MNRAS, 450, 3211

Baraffe, I., Chabrier, G., Allard, F., \& Hauschildt, P. H. 1998, A\&A, 337, 403

Barnes, J. R., Collier Cameron, A., Donati, J.-F., et al. 2005, MNRAS, 357, L1

Barrado Y Navascués, D. 2006, A\&A, 459, 511

Barrado y Navascués, D., Stauffer, J. R., \& Jayawardhana, R. 2004, ApJ, 614, 386

Borucki, W. J., Koch, D., Basri, G., et al. 2010, Science, 327, 977

Brandenburg, A., Moss, D., Rüdiger, G., \& Tuominen, I. 1991, Geophys. Astrophys. Fluid Dyn., 61, 179

Butters, O. W., West, R. G., Anderson, D. R., et al. 2010, A\&A, 520, L10

Collier Cameron, A. 2007, Astron. Nachr., 328, 1030

De Silva, G. M., D’Orazi, V., Melo, C., et al. 2013, MNRAS, 431, 1005

Distefano, E., Lanzafame, A. C., Lanza, A. F., et al. 2012, MNRAS, 421, 2774

Donahue, R. A., Saar, S. H., \& Baliunas, S. L. 1996, ApJ, 466, 384

Donahue, R. A., Dobson, A. K., \& Baliunas, S. L. 1997a, Sol. Phys., 171, 191

Donahue, R. A., Dobson, A. K., \& Baliunas, S. L. 1997b, Sol. Phys., 171, 211

Donati, J.-F., \& Collier Cameron, A. 1997, MNRAS, 291, 1

Donati, J.-F., Semel, M., Carter, B. D., Rees, D. E., \& Collier Cameron, A. 1997, MNRAS, 291, 658

Donati, J.-F., Mengel, M., Carter, B. D., et al. 2000, MNRAS, 316, 699

Feigelson, E. D., Lawson, W. A., \& Garmire, G. P. 2003, ApJ, 599, 1207

Ferreira Lopes, C. E., Leão, I. C., de Freitas, D. B., et al. 2015, A\&A, 583, A134
Gastine, T., Yadav, R. K., Morin, J., Reiners, A., \& Wicht, J. 2014, MNRAS, 438, L76

Gilliland, R. L., \& Fisher, R. 1985, PASP, 97, 285

Hussain, G. A. J. 2002, Astron. Nachr., 323, 349

Jeffers, S. V., \& Donati, J.-F. 2008, MNRAS, 390, 635

Kitchatinov, L. L., \& Rüdiger, G. 1999, A\&A, 344, 911

Kovacs, G. 1981, Ap\&SS, 78, 175

Küker, M., \& Rüdiger, G. 2011, Astron. Nachr., 332, 933

Küker, M., \& Stix, M. 2001, A\&A, 366, 668

Küker, M., Rüdiger, G., \& Kitchatinov, L. L. 2011, A\&A, 530, A48

Lanza, A. F., Rodonò, M., Pagano, I., Barge, P., \& A., L. 2003, A\&A, 403, 1135

Lanza, A. F., Rodonò, M., \& Pagano, I. 2004, A\&A, 425, 707

Lanza, A. F., Das Chagas, M. L., \& De Medeiros, J. R. 2014, A\&A, 564, A50

Lawson, W., \& Feigelson, E. D. 2001, in From Darkness to Light: Origin and Evolution of Young Stellar Clusters, eds. T. Montmerle, \& P. André, ASP Conf. Ser., 243, 591

López-Santiago, J., Montes, D., Crespo-Chacón, I., \& Fernández-Figueroa, M. J. 2006, ApJ, 643, 1160

Luhman, K. L., Stauffer, J. R., \& Mamajek, E. E. 2005, ApJ, 628, L69

Makarov, V. V. 2007, ApJS, 169, 105

Mamajek, E. E., Lawson, W. A., \& Feigelson, E. D. 1999, ApJ, 516, L77

Mamajek, E. E., Lawson, W. A., \& Feigelson, E. D. 2000, ApJ, 544, 356

Marsden, S. C., Carter, B. D., \& Donati, J.-F. 2005, 560, 799

Marsden, S. C., Donati, J.-F., Semel, M., Petit, P., \& Carter, B. D. 2006, MNRAS 370,468

Mentuch, E., Brandeker, A., van Kerkwijk, M. H., Jayawardhana, R., \& Hauschildt, P. H. 2008, ApJ, 689, 1127

Messina, S., \& Guinan, E. F. 2003, A\&A, 409, 1017

Messina, S., Desidera, S., Turatto, M., Lanzafame, A. C., \& Guinan, E. F. 2010, A\&A, 520, A15

Messina, S., Desidera, S., Lanzafame, A. C., Turatto, M., \& Guinan, E. F. 2011, A\&A, 532, A10

Moss, D., \& Brandenburg, A. 1995, Geophys. Astrophys. Fluid Dyn., 80, 229

Murphy, S. J., \& Lawson, W. A. 2015, MNRAS, 447, 1267

Murphy, S. J., Lawson, W. A., \& Bessell, M. S. 2013, MNRAS, 435, 1325

Parker, E. N. 1955, ApJ, 122, 293

Pecaut, M. J., \& Mamajek, E. E. 2013, ApJS, 208, 9

Perryman, M. A. C., Lindegren, L., Kovalevsky, J., et al. 1997, A\&A, 323, L49

Pojmanski, G. 1997, Acta Astron., 47, 467

Pollacco, D. L., Skillen, I., Collier Cameron, A., et al. 2006, PASP, 118, 1407

Reinhold, T., Reiners, A., \& Basri, G. 2013, A\&A, 560, A4

Schwarzenberg-Czerny, A. 1998, MNRAS, 301, 831

Siess, L., Dufour, E., \& Forestini, M. 2000, A\&A, 358, 593

Song, I., Zuckerman, B., \& Bessell, M. S. 2003, ApJ, 599, 342

Spada, F., Demarque, P., Kim, Y.-C., \& Sills, A. 2013, ApJ, 776, 87

Strassmeier, K. G. 2009, A\&ARv, 17, 251

Tamuz, O., Mazeh, T., \& Zucker, S. 2005, MNRAS, 356, 1466

Torres, C. A. O., Quast, G. R., da Silva, L., et al. 2006, A\&A, 460, 695

Torres, C. A. O., Quast, G. R., de La Reza, R., da Silva, L., \& Melo, C. H. F. 2001 , in Young Stars Near Earth: Progress and Prospects, eds. R. Jayawardhana, \& T. Greene, ASP Conf. Ser., 244, 43

Torres, C. A. O., Quast, G. R., Melo, C. H. F., \& Sterzik, M. F. 2008, Young Nearby Loose Associations, ed. B. Reipurth, 757

Waite, I. A., Marsden, S. C., Carter, B. D., et al. 2011, MNRAS, 413, 1949

Webb, R. A., Zuckerman, B., Platais, I., et al. 1999, ApJ, 512, L63

Zuckerman, B., \& Webb, R. A. 2000, ApJ, 535, 959 